\title{
EJECUCIÓN DE UN INSTRUMENTO MUSICAL Y ACTITUDES HACIA LA ESCUELA EN UNA MUESTRA DE ESTUDIANTES RUMANOS
}

\section{PERFORMANCE OF A MUSICAL INSTRUMENT AND ATTITUDES TOWARDS SCHOOL IN A SAMPLE OF ROMANIAN STUDENTS}

\author{
Cristina Cătălina Tudorie \\ Escuela de Arte Gymnasium "Victor Karpis" \\ cris.tudorie@gmail.com \\ https://orcid.org/0000-0002-1769-0322
}

\begin{abstract}
RESUMEN
El objetivo de este estudio fue investigar si existe diferencia significativa de actitudes hacia la escuela entre los estudiantes que estudian un instrumento musical y los que no lo hacen, en una muestra de 110 alumnos de entre 8 y 15 años del condado de Giurgiu, Rumania. Para la medición de la actitud hacia la escuela se utilizó el instrumento denominado School Attitude Assessment Survey. Una prueba $\mathrm{t}$ de Student para muestras independientes, mostró que existe una diferencia significativa entre ambos grupos. El estudiar la ejecución de un instrumento musical tuvo un impacto benéfico y promovió una actitud positiva hacia la escuela.
\end{abstract}

Palabras clave: actitud hacia la escuela, ejecución de un instrumento, educación musical

\section{ABSTRACT}

The aim of this study was to investigate whether there is a significant difference in attitudes towards school between students who study a musical instrument and those who do not, in a sample of 110 8-15 years old students from Giurgiu County, Romania. The School Attitude Assessment Survey was used to measure the attitudes towards school. A Student's t-test for independent samples showed that there is a significant difference between both groups. Studying the performance of a musical instrument had a beneficial impact and promoted a positive attitude towards school.

Keywords: attitude towards school, playing an instrument, musical education 


\section{Introducción}

Como fenómeno complejo, arte y ciencia al mismo tiempo, al tener un lenguaje con un sistema específico de símbolos, la música desarrolla una comunicación expresiva que se entrelaza con el proceso del conocimiento (Iamandescu, 2011).

La música ha sido considerada un medio esencial para el cultivo espiritual del hombre, para perfeccionar una personalidad equilibrada y armoniosa, acompañando al hombre en su vida íntima, psicológica, religiosa, moral, cívica y social (Vasile, 2004). Influye sobre la autoestima y las aspiraciones de las personas y puede mejorar el comportamiento y las habilidades sociales, la alfabetización y el lenguaje (Henley, 2011). Además, si es adquirida solo como fenómeno artístico en sí misma, sigue siendo un valor externo al hombre, pero percibida a nivel espiritual, se convierte en parte de su universo íntimo, transformándolo y edificándolo.

Sacks (2009) señala que la música no tiene un centro en el cerebro humano, sino que involucra numerosas redes, diseminadas por todo el cerebro, que activan diversas áreas del cerebro. Actúa directa y simultáneamente sobre los principales centros del sistema nervioso. Levitin (2013) agrega que el cerebro utiliza la segregación funcional para procesar la música y utiliza un sistema de detectores con determinadas características, cuya misión es analizar las cualidades específicas del sonido musical, como el tono, el tempo y el timbre, entre otros.

Por otro lado, un estudio realizado en una escuela secundaria en Shanghai (Ho y Law, 2006) reveló que existen vínculos ricos y variados entre los contextos sociales de los estudiantes y sus experiencias musicales, mostrando que la mayoría de los estudiantes aprecian la música como expresión de sentimientos. $\mathrm{Su}$ experiencia musical y de aprendizaje estaba relacionada con los medios de comunicación, pero también con la vida escolar o familiar.

La estimulación auditiva temprana y la experiencia musical desde la infancia son importantes para obtener actuaciones superiores en el campo musical. Ullal-Gupta et al. (2013) muestran que el entorno auditivo intrauterino juega un papel clave en el posterior modelado del desarrollo auditivo y las preferencias musicales del niño. Existe evidencia de que los sonidos generados desde el exterior y el interior del entorno uterino influyen en el desarrollo del feto. Tal experiencia auditiva prenatal puede establecer la trayectoria para el desarrollo de un intelecto musical.

Así mismo, se ha observado que la música puede tener efectos positivos en las habilidades comunicativas de los niños con dificultades de aprendizaje, al favorecer el desarrollo de una memoria que aumenta sus capacidades cognitivas, observables particularmente en el incremento de las habilidades comunicativas de los niños con trastornos del espectro autista, con trastornos emocionales o con psicopatologías (Perret, 2004).

Se ha estudiado el efecto que tiene la musica sobre el rendimiento academico en las clases de matematicas. Los resultados mostraron que la música, en general, tiene un efecto positivo. Los estudiantes experimentaron mejoras en las calificaciones de matemáticas los días que escucharon música en la clase de matemáticas. Además, el comportamiento de estos niños cambió de manera positiva. Los estudiantes informaron que se sentían diferentes y tenían más 


\section{EJECUCIÓN DE UN INSTRUMENTO MUSICAL}

éxito en matemáticas en el aula cuando se ejecutaba música clásica (McKeever, 2017).

\section{La impacto de la música en el cuerpo humano}

La inclusión de componentes del ritmo motor en la musicoterapia ha conducido a mejoras significativas en la atención selectiva, el habla y la adquisición en niños con autismo (Janzen y Thaut, 2018).

En un estudio realizado en Louisiana (Bowers et al., 2019) en 62 centros de cuidado infantil, se probó el efecto de la música en las horas de siesta. La mayoría de los niños solían necesitar más de 30 minutos para conciliar el sueño. Los niños de estos centros de cuidados dormían con música. La mayoría de los profesores prefería utilizar la misma música todos los días. Preferían la música religiosa o la música instrumental. Los maestros descubrieron que la música tenía un efecto calmante en los niños de ese centro de cuidado.

La investigación médica y científica actual (Fauble, 2017) destaca que la música y el sonido son algunas de las herramientas más efectivas que se pueden utilizar para facilitar la recuperación del paciente de una cirugía, trauma y enfermedad. Así, la música puede aliviar el sufrimiento mental, emocional $\mathrm{y}$ físico.

La música en los primeros años de vida está asociada con cambios en la función cerebral y con la mejora de las habilidades del lenguaje. La formación musical compensa la disminución del procesamiento auditivo del cerebro que acompaña al envejecimiento normal de los seres humanos. La robusta neuroplasticidad conferida por la formación musical no está limitada por la edad; puede servir como un medio eficaz para consolidar el deterioro de las habilidades del habla a lo largo de la vida (Bidelman y Alain, 2015).

Rickard y McFerran (2012) descubrieron que la música puede promover la salud y el funcionamiento mental, tanto en términos del desarrollo cognitivo de los bebés prematuros, el establecimiento de la identidad y el bienestar emocional en los adolescentes, como en el desarrollo de las funciones cerebrales en los adultos y el deterioro cognitivo en los pacientes con demencia.

Por otro lado, se observó un aumento en el coeficiente intelectual en preescolares involucrados en la composición musical (Schellenberg, 2004). Otros estudios mostraron los beneficios de la educación musical para el desarrollo de las funciones cognitivas, incluida la memoria visual y verbal (Rickard et al., 2010).

\section{La música y el desarrollo de la personalidad}

La función comunicativa de la música se completa con la expresiva, cognitiva, estética y educativa, ofreciendo múltiples formas de desarrollar al ser humano, con influencias beneficiosas sobre la actividad psicológica y sobre todo el cuerpo humano (Iamandescu, 2011). El niño nace con un determinado equipamiento genético que, en general, no favorece por igual todos los rasgos de carácter esenciales en la composición de una personalidad desarrollada armoniosamente. En el ámbito de la personalidad normal, se encontró que la inteligencia innata de un individuo se puede desarrollar educando esa función en casi un 10\% (Iamandescu, 2011). Cury (2018) sostiene que la música puede influir en los niños en su hiperactividad, 
modulando el ritmo del pensamiento y estabilizando las emociones.

Nyklfaek (citado en O'Gorman, 2007) ha observado el poder de la música sobre la condición humana, identificando conexiones entre el estado fisiológico y emocional, en un estudio que muestra que la música genera estados de felicidad, tristeza, serenidad o agitación. La gente escucha música solo como fondo para otras actividades, para la regulación emocional o para conocerla. Un estudio (Chamorro-Premuzic y Furnham, 2007) mostró que las personas abiertas, extrovertidas y con un coeficiente intelectual alto tienden a usar la música de manera racional y cognitiva, en contraste con las que son introvertidas, neuróticas y no conscientes, que usan la música para regular sus emociones y para cambiar y mejorar su estado de ánimo, enfocándose más en el contenido que en la estructura musical.

Con el desarrollo de la musicalidad, el hábito de cantar tiene un efecto positivo sobre otros rasgos de personalidad en la formación de los niños pequeños. Así, la espontaneidad y la comunicabilidad sustituyen a la timidez y al encarcelamiento, habituales en esta etapa. La disciplina colectiva, la atención distributiva, la participación emocional en la ejecución de la canción, con la movilización de componentes importantes de la psique del niño, la memoria compleja, todo ello requerido simultáneamente sobre el texto, el ritmo y la armonía durante el canto grupal o individual, son ventajas conocidas por todos los profesores. Por lo tanto, la educación musical en los estudiantes tiene beneficios creativos, sociales y cognitivos, más adelante en la vida de los adolescentes (Pitts, 2017).

En un estudio realizado en 2012 y 2013 en Venezuela (Alemán et al., 2017) sobre un grupo de 2914 niños del Sistema Nacional Juvenil y Orquestal para niños de entre 6 y 14 años, se observó luego de la intervención que se mejoró el autocontrol de los niños y se redujeron las dificultades de comportamiento, particularmente el nivel de comportamiento agresivo.

\section{El impacto de estudiar un instrumento musical en los niños}

Escuchar música, y en particular ejecutar un instrumento, fortalece o modifica los circuitos neuronales, incluida la densidad de conexiones dendríticas en la corteza auditiva primaria. La parte frontal del cuerpo calloso, el "puente" de fibras que conecta los hemisferios cerebrales, está visiblemente más desarrollada en los músicos que en los que no son músicos, lo que permite que los mensajes pasen por el cerebro por rutas más diversas, especialmente para los músicos que han comenzado a entrenar temprano (Levitin, 2013).

La diferencia más obvia entre escuchar música y producirla es que esta última requiere habilidades motoras especiales, que son controladas por ambos hemisferios del cerebro, combinando precisión lingüística y matemática, en la que el hemisferio izquierdo está más involucrado, con contenido creativo, en el que predomina el hemisferio derecho. Además, un grupo de investigadores de la Universidad de Harvard ha demostrado que los niños que estudian la ejecución de un instrumento musical durante al menos tres años superan a otros niños incluso en otras dos habilidades: habilidad verbal, medida con una prueba de vocabulario, y la visual, observable mediante el fácil reconocimiento de estructuras (Iamandescu, 2011). 


\section{EJECUCIÓN DE UN INSTRUMENTO MUSICAL}

Los niños que crecen escuchando música, tarareando canciones o ejecutando un instrumento son mucho más receptivos y acumulan conocimientos más rápidamente, porque la música usa estructuras cerebrales y circuitos neuronales que otras actividades no involucran. El proceso por el cual uno se convierte en músico, ya sea compositor o intérprete, requiere el cultivo de valores especificos: diligencia, paciencia, motivación y perseverancia (Levitin, 2013).

En Alemania, Hille y Schupp (2015) demostraron que las habilidades de los niños que están expuestos a lecciones de música y a estudiar un instrumento son asombrosas. El progreso que hacen, las habilidades que desarrollan, las implicaciones a medio y largo plazos, la disciplina que aprenden de forma natural, la creatividad, la atención al detalle, la paciencia y la confianza en sí mismos, son solo algunas de las ventajas que adquieren los niños que estudian un instrumento. El estudio también enfatiza que quienes aprenden música tienen mejores habilidades cognitivas y calificaciones en la escuela, son más conscientes, más abiertos y más ambiciosos. La música desarrolla el conocimiento y la mente más que los deportes, el teatro o la danza. Además, estudiar un instrumento musical ayuda a aprender mejor un idioma extranjero e interpretar las emociones de los demás. Una de las razones por las que la música puede traer tantos beneficios al cerebro es la intersección de los sistemas emocional y de conocimiento involucrados en el acto emocional.

\section{La influencia del entorno familiar en el desarrollo musical de los niños}

Mombourquette (2007) sostiene que existe una relación entre algunos de los tipos de participación de los padres y el desempeño de los estudiantes, su rendimiento académico y su actitud hacia la escuela.

Un estudio con un grupo de 257 estudiantes mostró que el apoyo de los padres tiene efectos favorables sobre el desarrollo de los niños y sus resultados son mucho mejores en el campo de la música (Davidson et al., 1996).

Un estudio (Comeau et al., 2015) comparó a 50 niños chinos y 100 niños caucásicos en América del Norte de 6 a 17 años que estaban aprendiendo a ejecutar el piano para comprender la ética laboral, la motivación y la influencia de los padres. Se descubrió que los estudiantes chinos practicaron casi el doble en el piano para lograr la interpretación deseada, en comparación con los caucásicos en América del Norte. Los padres de los estudiantes chinos participaron en las lecciones de piano de sus hijos. Su presencia motivó a sus hijos a trabajar más en el piano. Este estudio ayuda a comprender mejor la ética de trabajo, la motivación y el apoyo de los padres de los estudiantes de piano chinos, lo que puede ayudar a explicar el éxito de los músicos asiáticos.

\section{Actitud hacia la escuela de los estudiantes que estudian un instrumento musical}

Se ha mostrado que la dinámica interpersonal de las relaciones alumno-padre y alumno-profesor representa una fuerte influencia en el aprendizaje escolar, en los alumnos que estudian un instrumento musical (Creech y Hallam, 2010).

Un estudio realizado en EE. UU. (Hopkins et al., 2017) mostró la relación entre un alto nivel de logro musical, mayores oportunidades de aprendizaje y la promoción de valores como la disciplina, la perseverancia y las actitudes 


\section{TUDORIE}

positivas hacia el trabajo en un grupo de estudiantes de quinto grado. Por otro lado, se encontró que el programa de educación musical de Harmonix Music Systems ayuda a los estudiantes a componer musica y promueve el aprendizaje cooperativo en el aula (Richmond et al., 2016).

\section{Metodología}

El estudio utilizó una metodología cuantitativa, descriptiva, comparativa, de campo y transversal.

\section{Participantes}

La muestra incluyó a 110 alumnos de entre 8 a 15 años, de ambos sexos, del condado de Giurgiu, Rumania.

Los estudiantes se dividieron en dos categorías: los que estudiaban un instrumento musical $(n=50,45.5 \%)$ y los que no lo hacían $(n=60,54.5 \%)$.

Se compiló una lista de todos los miembros de la muestra y se asignó aleatoriamente un número a cada participante del estudio, con el fin de preservar su confidencialidad. La participación en la prueba respondió a los números recibidos y no a los nombres.

\section{Instrumento}

La herramienta utilizada para medir la actitud hacia la escuela fue el School Attitude Assessment Survey, desarrolla- do, revisado y validado por McCoach (2002), y traducido y validado al rumano por Popa (2011).

De la escala Likert de siete puntos se eliminó el nivel 4 que, en el instrumento original, corresponde al enunciado ni de acuerdo ni en desacuerdo, para eliminar el nivel de neutralidad (Popa, 2011). Por lo tanto, las opciones de respuesta quedaron como sigue: totalmente en desacuerdo, en desacuerdo, levemente en desacuerdo, levemente de acuerdo, de acuerdo, totalmente de acuerdo.

El cuestionario tiene un coeficiente de confiabilidad alfa de .9431 .

\section{Resultados}

Aunque la edad de los estudiantes encuestados se hallaba entre los 8 y los 15 años, la gran mayoría de ellos $(n=$ $91,82,65 \%$ ) se ubicó entre los 11 y los 14 años.

\section{Análisis descriptivo}

Se obtuvo el valor mínimo y máximo, la media y la desviación estándar de la escala general y de cada una de las subescalas. Para la actitud general hacia la escuela, de todos los estudiantes que participaron en el estudio, se obtuvo un valor mínimo de 2,66 y uno máximo de $6,00(M=4,96, D E=0,76)$. En la Tabla 1 se presentan los resultados de cada una de las subescalas.

\section{Tabla 1}

Análisis descriptivo de la actitud general hacia la escuela y sus subescalas

\begin{tabular}{lcccc}
\hline \multicolumn{1}{c}{ Subescala } & Mínimo & Máximo & $M$ & $D E$ \\
\hline Autopercepción académica & 2,29 & 6,00 & 4,88 & 0,90 \\
Actitud hacia profesores y compañeros & 1,86 & 6,00 & 5,08 & 0,84 \\
Actitud hacia la escuela & 2,20 & 6,00 & 4,99 & 1,01 \\
Importancia de los objetivos & 3,17 & 6,00 & 5,41 & 0,64 \\
Motivación y autorregulación & 1,50 & 6,00 & 4,64 & 1,09 \\
\hline
\end{tabular}




\section{EJECUCIÓN DE UN INSTRUMENTO MUSICAL}

Un analisis por ítems permitió observar que la afirmación con el promedio más alto fue "quiero sacar buenas notas en la escuela" (ítem 15), con un promedio de 5,59 $(D E=0,78)$, seguido de "es importante para mí hacerlo bien en la escuela" (ítem 29), con un promedio de $5,45(D E=0,88)$, y de "si me va bien en la escuela esto es importante para mi futura carrera" (ítem 18), con un promedio de 5,51 $(D E=0,86)$. El enunciado que registró el promedio más bajo es "dedico mucho tiempo a la tarea" (ítem 30), con un promedio de 4,16 $(D E=1,55)$. Esta conclusión que caracterizó a los estudiantes no puede pasarse por alto, ya que la afirmación "quiero sacar buenas notas en la escuela" fue el elemento con el promedio más alto y "dedico mucho tiempo a la tarea" el elemento con el promedio más bajo en este estudio.

\section{Análisis comparativo}

Se comparon las dos categorías de estudiantes, los que estudian la ejecución de un instrumento musical (Grupo I) y los que no lo hacen (Grupo II), para observar las diferencias en cada una de las subescalas de actitudes hacia la escuela. Finalmente, se comparó la actitud general media hacia la escuela entre los dos grupos de estudiantes.

Así, al aplicar la prueba $t$, se concluyó que existe una diferencia significativa en la actitud hacia la escuela entre los grupos del estudio, pues la escala general de actitud hacia la escuela $\left(t_{(108)}\right.$ $=4,160, p=, 000)$ mostró un promedio de 5,27 $(D E=0,68)$ para los estudiantes que conformaban el Grupo I y 4,70 (DE $=0,73)$ para los del Grupo II. La Tabla 2 incluye esta información junto con la de cada una de las subescalas.

\section{Tabla 2}

Análisis comparativo de la actitud hacia la escuela entre los dos grupos

\begin{tabular}{lccccc}
\hline \multirow{2}{*}{\multicolumn{1}{c}{ Subescala }} & \multicolumn{3}{c}{ Media } & & \\
\cline { 2 - 3 } & Grupo I & Grupo II & & \\
\hline Autopercepción académica & 5,24 & 4,59 & & 4,157 &, 000 \\
Actitud hacia profesores y compañeros & 5,25 & 4,94 & 1,197 &, 052 \\
Actitud hacia la escuela & 5,38 & 4,68 & 3,942 &, 000 \\
Importancia dada a los objetivos & 5,60 & 5,26 & 2,837 &, 005 \\
Motivación y autorregulación & 5,05 & 4,29 & 3,964 &, 000 \\
Escala general & 5,27 & 4,70 & & 4,160 &, 000 \\
\hline
\end{tabular}

Se observaron diferencias muy significativas entre los grupos en cuatro subescalas: autopercepción académica, actitud hacia la escuela, importancia dada a los objetivos, y motivación y autorregulación. Todos mostraron una actitud significativamente favorable, hacia la escuela, por parte del grupo que estudia la ejecución de un instrumento musical.
Autopercepción académica. En el enunciado "la escuela es fácil para mí" (ítem 13), el 25\% de los encuestados de ambas muestras estuvo totalmente de acuerdo, lo que significa que el $75 \%$ de los estudiantes enfrentó diversas dificultades de aprendizaje. En el enunciado "puedo entender procesos complejos en la escuela" (ítem 20), solo el 23\% de los encuestados estuvo de acuerdo; los 
estudiantes del Grupo II incluso respondieron en un porcentaje menor, de solo el $11 \%$, con totalmente de acuerdo.

Actitud hacia profesores y compañeros. En el enunciado "me gustan mis profesores" (ítem 14) y "muchos de los profesores de mi escuela son buenos profesores" (ítem 31), el 66\% de los encuestados de ambas muestras estuvo totalmente de acuerdo, sin respuestas en desacuerdo o totalmente en desacuerdo.

Actitud hacia la escuela. La afirmación "esta es una buena escuela" (ítem 7) mostró respuestas de totalmente de acuerdo entre el $90 \%$ de los encuestados del Grupo I, pero la afirmación "estoy orgulloso de esta escuela" (ítem 23) registró la puntuación más baja de esta investigación, porque nueve de los estudiantes del Grupo II $(15 \%)$ manifestó estar totalmente en desacuerdo.

Importancia dada a los objetivos. Para las declaraciones "es importante sacar buenas notas en la escuela" (ítem 25), "si me va bien en la escuela, esto es importante para las metas de mi futura carrera" (ítem 18) y "quiero hacer lo que mejor puedo en la escuela" (ítem 28), el $92 \%$ de los encuestados del Grupo I estuvo de acuerdo y totalmente de acuerdo, en comparación con el $65 \%$ de los del Grupo II.

Con el enunciado "quiero sacar buenas notas en la escuela" (ítem 15), el 91\% de los estudiantes del Grupo I estuvo de acuerdo y totalmente de acuerdo, en comparación con solo el $65 \%$ de los estudiantes del Grupo II.

Motivación y autorregulación. En las afirmaciones "reviso mi tarea antes de presentarla" (ítem 4), "dedico mucho tiempo a la tarea" (ítem 30) y "me esfuerzo mucho en la tarea" (ítem
33 ), solo el $16 \%$ de los encuestados del Grupo II estuvo totalmente de acuerdo, lo que significa que el $84 \%$ restante de los estudiantes no estuvo interesado en hacer los deberes. Además, en la afirmación "trabajo duro en la escuela" (ítem 8), solo el 28\% de los encuestados del Grupo II estuvo totalmente de acuerdo, en tanto el $72 \%$ restante de los encuestados no hizo un esfuerzo sostenido para obtener buenos resultados escolares.

Diferencias de género. Cuando se comparó la actitud hacia la escuela entre niños y niñas, se pudo observar que las niñas mostraron una actitud general más favorable hacia la escuela que los niños $\left(t_{(108)}=3,428, p=, 001\right)$. Las niñas registraron un promedio de la actitud general hacia la escuela de 5,12 (DE = $0,68)$, mientras que los niños registraron un promedio de 4,62 $(D E=0,83)$. Cuando se analizaron las cinco subvariables, se observó que hubo puntuaciones significativamente más altas de las niñas en comparación con los niños en las siguientes: autopercepción académica $\left.t_{(108)}=2,793, p=, 006\right)$, donde los niños registraron un promedio de 4,55 $(D E=$ $0,92)$, mientras que las niñas registraron uno de 5,04 $(D E=0,84)$; actitud hacia profesores y compañeros $\left(t_{(108)}=2,440\right.$, $p=, 016)$, donde los niños registraron un promedio de $4,81(D E=0,94)$ y las niñas uno de 5,21 $(D E=0,76)$; actitud hacia la escuela $\left(t_{(108)}=2,883, p=, 005\right)$, donde los niños registraron un promedio de 4,61 $(D E=1,12)$, mientras que las niñas registraron uno de 5,18 $(D E=$ $0,90)$; importancia dada a los objetivos $\left.t_{(108)}=2,992, p=, 003\right)$, donde los niños registraron un promedio de $5,16(D E$ $=0,75)$ y las niñas uno de $5,54(D E=$ $0,54)$; y motivación y autorregulación $\left.t_{(108)}=2,995, p=, 004\right)$, donde los niños 


\section{EJECUCIÓN DE UN INSTRUMENTO MUSICAL}

registraron un promedio de $4.21(D E$ $=1,28)$ y las niñas uno de $4.84(D E=$ $0,93)$.

\section{Discusión}

Dentro de la subescala autopercepción académica, se observó que los estudiantes que estudian la ejecución de un instrumento musical pueden comprender procesos complejos en la escuela en un $12 \%$ más que quienes no lo hacen. Asimismo, en la subescala motivación y autorregulación, se observó que el interés por cumplir con los deberes y por hacer un esfuerzo sostenido para obtener buenos resultados escolares fue $64 \%$ mayor en los estudiantes que estudian un instrumento musical. En cuanto a la subescala importancia que se le da a las metas y objetivos, el $92 \%$ de los encuestados de la escuela de música enfatizó con puntaje máximo que es importante y quiere obtener buenas calificaciones en la escuela, con el fin de lograr los objetivos de su futura carrera.

La música es un buen ejemplo de una actividad que influye en otros aspectos de la vida que requieren voluntad, disciplina, dedicación, tiempo, estudio intenso, trabajo e incluso competitividad. Para quienes quieren alcanzar un alto nivel, desarrollan habilidades de comunicación, comprensión, conciencia y creatividad y cultivan la responsabilidad, la perseverancia, la dignidad, la armonía interior, el respeto y la exigencia. Además, la música influye benéficamente en el comportamiento y la actitud (Aldea y Munteanu, 2001). Por medio de la música se entrena permanentemente el pensamiento independiente y la fantasía creativa. Gracias a ella se reduce en los niños la contención y la inhibición, a la vez que aumentan continuamente la capacidad del niño para la concentración y coordinación (Niedermaier, 1999).

Las actitudes de los estudiantes hacia la escuela están influenciadas en gran medida por la percepción que tiene el niño de su propia capacidad. Su autoeficacia le permite trabajar para lograr sus metas (Valeski y Stipek, 2001), al igual que la percepción del niño respecto de la actitud del maestro hacia él.

Se puede concluir que existe una diferencia estadísticamente significativa en la actitud hacia la escuela entre los dos grupos, siendo el estudio de la ejecución de un instrumento musical un predictor positivo significativo de la actitud de los estudiantes hacia la escuela.

Asimismo, la actitud hacia la escuela está influenciada por la sociabilidad y motivación del niño, por su respeto y autoestima (Brand, 2004). La música y el entorno escolar o sociocultural en el que se estudia puede producir cambios relacionados con el entorno interno del adolescente transformando sus representaciones de su mundo interior, especialmente el emocional (Rutter, 1980).

Los niños cuyos padres participan en lecciones con ellos están más motivados para estudiar el instrumento y muestran un mayor interés por la música.

La dinámica interpersonal de las relaciones alumno-padre y alumno-profesor es un predictor significativo de la actitud hacia la escuela.

La interpretación de un instrumento musical es un predictor significativo de las actitudes de los estudiantes hacia la escuela, ofrece mayores oportunidades de aprendizaje, desarrollo, pero también promociona valores tales como la perseverancia, la disciplina y una actitud positiva hacia el trabajo en general. 


\section{TUDORIE}

\section{Referencias}

Aldea, G. y Munteanu, G. (2001). Didactica educației muzicale în învățământul primar. Didactică și Pedagogică.

Alemán, X., Duryea, S., Guerra, N. G., McEwan, P. J., Muñoz, R., Stampini, M. y Williamson, A. A. (2017). The effects of musical training on child development: A randomized trial of El Sistema in Venezuela. Prevention Science, 18(7), 865-878. https://doi.org/10.1007/s11121-016-0727-3

Bidelman, G. M. y Alain C. (2015). Musical training orchestrates coordinated neuroplasticity în auditory brainstem and cortex to counteract age-related declines in categorial vowel perception. Journal of Neuroscience, 35(3), 1240-1249. https://doi.org/10.1523/JNEUROSCI.3292-14.2015

Bowers, J. P., Cassellberry, J. J., Isbell, D., Kyakuwa, J., Li, Y., Mercado, E. M. y Wallace, E. M. (2019). A descriptive study of the use of music during naptime in Louisiana child care centers. Journal of Research in Childhood Education, 33(2), 212-224. https://doi.org/10.1080/02568543.201 9.1577770

Brand, M. (2004). Collectivistic versus individualistic cultures: A comparation of American, Australian and Chinese music education, student's self-esteem. Music Education Research, 6(1), 57-66. https:// doi.org/10.1080/1461380032000182830

Chamorro-Premuzic, T. y Furnham, A. (2007). Personality and music: Can traits explain how people use music in everyday life? British Journal of Psychology, 98(2), 175-185. https://doi. org $/ 10.1348 / 000712606$ X111177

Comeau, G., Huta, V. y Liu, Y. (2015). Work ethic, motivation, and parental influences in Chinese and North American children learning to play the piano. International Journal of Music Education, 33(2), 181-194. https://doi.org/10.1177/0255761413516062

Creech, A. y Hallam, S. (2010). Learning a musical instrument: The influence of interpersonal interaction on outcomes for school-aged pupils. Psychology of Music, 39(1), 102-122. https://doi. org $/ 10.1177 / 0305735610370222$

Cury, A. (2018). Părinți străluciți, profesori fascinanți: Cum să formăm tineri fericiți și străluciți. For You.

Davidson, J. W., Howe, M. J. A., Moore, D. G. y Sloboda, J. A. (1996). The role of parental influences in the development of musical performance. British Journal of Development Psychology, 14, 399-412. https://doi.org/10.1111/j.2044-835X.1996.tb00714.x

Fauble, L. (2017). From Neanderthal to neuroscience: Healing with sound and voice. Voice and Speech Review, 11(1), 72-86. https://doi.org/10.1080/23268263.2017.1370838

Henley, D. (2011). Music education in England: A review by Darren Henley for the Department for Education and the Department for Culture, Media and Sport. Departament for Education.

Hille, A. y Schupp, J. (2015). How learning a musical instrument affects the development of skills. Economics of Education Review, 44, 56-82. https://doi.org/10.1016/j.econorev.2014.10.007

Ho, W. C. y Law, W. W. (2006). Students' music experiences, society and culture: Music education in Shanghai, China. Music Education Research, 8(1), 47-64. https://doi. org/10.1080/14613800600570728

Hopkins, M., Provenzano, A. M. y Spencer, M. S. (2017). Benefits, challenges, characteristics and instructional approaches in an El Sistema inspired after-school string program developed as a university-school partnership in the United States. International Journal of Music Education, 35(2), 239-258. https://doi.org/10.1177/0255761416659509

Iamandescu, I. B. (2011). Muzicoterapia receptivă: Premise psihologice şi neurofiziologice, aplicaţii profilactice şi terapeutice. Fundației “Andrei Șaguna”.

Janzen, T. B. y Thaut, M. H. (2018). Rethinking the role of music in the neurodevelopment of autism spectrum disorder. Music \& Science, 1. https://doi.org/10.1177/2059204318769639 


\section{EJECUCIÓN DE UN INSTRUMENTO MUSICAL}

Levitin, D. J. (2013). Creierul nostru muzical: Știința unei eterne obsesii. Humanitas.

McCoach, D. B. (2002). A validation study of the school attitude assessment survey. Measurement and Evaluation in Counseling and Development, 35(2), 66-77. https://doi.org/10.1080/07481756.2002.1 2069050

McKeever, A. C. (2017). The effects of classical music on children with learning disabilities in a sixth grade mathematics classroom (Publicación No 10287634) [Tesis de maestría, Rowan University]. ProQuest Dissertations and Theses Global.

Mombourquette, C. P. (2007). A study of the relationship between the type of parent involement and high school student engagement, academic achievement, attendance and attitude toward school (Publicación No 3258724) [Tesis doctoral, The University of Montana). ProQuest Dissertations and Theses Global.

Niedermaier, A. (1999). Educație muzicală modernă: Concepția pedagogică şi instrumentariul Orff. Hora.

O'Gorman, S. (2007). Infant-directed singing in neonatal and paedriatic intensive care. Australian end NewZealand Journal of Family Therapy, 28(2), 100-108. https://doi.org/10.1375/anft.28.2.100

Perret, D. (2004). Roots of musicality: on neuro-musical theresholds and new evidence for bridges between musical expression end 'inner growth'. Music Education Research, 6(3), 327-342. https:// doi.org/10.1080/1461380042000281767

Pitts, S. (2017). What is music education for? Understanding and fostering routes into lifelong musical engagement. Music Education Research, 19(2), 160-168. https://doi.org/10.1080/14613808.2016.1166196

Popa, D. (2011). Climatul familial și atitudinea față de școală a elevilor de nivel liceal [Tesis de maestría, Universidad de Montemorelos]. DSpace. http://dspace.biblioteca.um.edu.mx/xmlui/ handle/20.500.11972/1229

Richmond, J., McLachlan, N. M., Ainley, M. y Osborne, M. (2016). Engagement and skill development through an innovative classroom music program. International Journal of Music Education, 34(2), 143-160. https://doi.org/10.1177/0255761415584289

Rickard, N. S. y McFerran, K. (Eds.). (2012). Lifelong engagement with music: Benefits for mental health and well-being. Nova Science.

Rickard, N. S., Vasquez, J. T., Murphy, F., Gill, A. y Toukhsati, S. R. (2010). Benefits of a classroom based instrumental music program on verbal memory of primary school children: A longitudinal study. Australian Journal of Music Education, 2010(1), 36-47. https://search.informit.com.au/ documentSummary; $\mathrm{dn}=691191050436949 ;$ res $=$ IELHSS

Rutter, M. (1980). Changing youth in a changing society: Patterns of adolescent development and disorder. Harvard University Press.

Sacks, O. (2009). Muzicofilia: Povestiri despre muzică şi creier. Humanitas.

Schellenberg, E. G. (2004). Music lessons enhance IQ. Psychological Science, 15(8), 511-514. https://doi. org/10.1111/0956-7976.2004.00711.x

Ullal-Gupta, S., Vanden Bosch der Nederlanden, C. M., Tichko, P., Lahav, A. y Hannon, E. E. (2013). Linking prenatal experience to the emerging musical mind. Frontiers in Systems Neuroscience, 7, 48. https://doi.org/10.3389/fnsys.2013.00048

Valeski, T. N. y Stipek, D. J. (2001). Young children's feelings about school. Child Development, 72(4), 1198-1213. https://doi.org/10.1111/1467-8624.00342

Vasile, V. (2004). Metodica educației muzicale. Editura Muzicală.

Recibido: 15 de abril de 2020

Revisado: 15 de mayo de 2020

Aceptado: 22 de mayo de 2020 